\title{
Comparative analysis of simulation environments for a virtual testing ground to measure vibration and acoustic emissions of designed flying objects
}

\author{
Kristina Abramkina ${ }^{1}$, Elena Zhilenkova ${ }^{2, *}$, and Vladislav Borisenko ${ }^{2}$ \\ ${ }^{1}$ Saint Petersburg State Marine Technical University, 190121, St. Petersburg, Russian Federation \\ ${ }^{2}$ Peter the Great St. Petersburg Polytechnic University, 195251, St. Petersburg, Russia
}

\begin{abstract}
The article deals with an important issue of creating virtual test grounds. The problem of measuring vibrations and acoustic emissions of designed objects of the aircraft industry, etc is studied. Thus, for example, one of the important tests of the coaxial helicopter at the development stage is the assessment of the impact of manufacturing tolerances on fuselage vibrations. The general problems of modeling and control of the acoustic field, the measurement of the acoustic emission of objects are considered. The research results can be used to solve the problems of creating virtual test grounds and other digital systems, where the assessment of the behavior of a complex object is made on the basis of its digital twin or, in general, on a mathematical or computer model. The introduction of such systems can significantly reduce financial costs when creating a product, increase quality of designed systems and predict their behavior under certain conditions without expensive testing.
\end{abstract}

\section{Introduction}

To undertake the research, the software environments for the development of Matlab and COMSOL and examples of projects carried out in them are considered. The objective of the study is to compare the parameters and technical characteristics that are important for modeling the acoustic field. For this purpose, in the Matlab development environment, it is necessary to examine the processes of the formation of an ultrasonic field, obtain a calculation of perturbations and failures of lattice elements from ultrasound sensors. Moreover, the structure and calculation of the total acoustic pressure in the Comsol environment should also be analyzed.

Let's us consider the acoustic levitation field in a standing wave. It can be used to levitate objects with a diameter less than half $(\lambda)$. The effect is achieved with a sound emitter and reflector (usually a concave shape for better focusing) or two or more sound emitters. In the space between the reflector and the emitter, interference occurs between the emitted and reflected waves of the same frequency and phase. Interference of radiated waves from 2 or more sound emitters may also occur. Weakening radiated waves are amplified by counter-

\footnotetext{
* Corresponding author: zhilenkovanton@gmail.com
} 
propagating waves from a reflector or a second sound emitter, thus creating a standing wave levitation field.

If we use 4 sound emitters, we can get 2 intersecting standing wave levitation fields to control space along 3 axes.

The transverse radiation force (radial force) is a pressure force acting in the transverse direction relative to the primary force. This force arises due to the fact that the object in the field is an obstacle, therefore the environment flows around the particle. This effect is limited to the area around the particle. 3]:

To determine the pressure of acoustic radiation on a sphere, the King's theory is used [1-

$$
\delta p=p-p_{0=} p_{0} \dot{\phi}+\frac{1}{2} \frac{p_{0}}{c^{2}} \phi^{2}-\frac{1}{2} p_{0} q^{2},
$$

where $\dot{\phi}$ is a velocity potential

$$
\dot{\phi}=\frac{D \phi}{D t}-\dot{\xi} \frac{d \phi}{d x}-\dot{\eta} \frac{d \phi}{d y}-\dot{\zeta} \frac{d \phi}{d z}=\frac{D \phi}{D t}+u \dot{\xi}+v \dot{\eta}+w \dot{\zeta}
$$

$\mathrm{q}^{2} / \mathrm{c}^{2}-$ final result: $\mathrm{c}^{2}-$ constant and $\frac{d p}{d \rho}, d \rho-$ density of the medium.

$\dot{\xi}, \dot{\eta}, \dot{\zeta}$ give second-order contributions to pressure variations over the boundary and are of the same magnitude order.

So the flow velocity around the particle decreases with increasing distance $[2,4]$.

$$
\dot{\Phi}-\int \frac{d p}{p}=\frac{v^{2}}{2}
$$

This creates additional pressure that increases with increasing distance. This leads to particle stabilization.

The change in pressure depends on the distance between the emitter and the levitated object [3].

Acoustic power for a small solid is calculated according to the following formula (4):

$$
8 \pi r_{s}\left(k r_{s}\right) \bar{E} \sin (2 k h) f\left(\frac{p_{0}}{p_{s}}\right),
$$

where $\mathrm{k}$ is the number of waves,

$r_{\mathrm{s}}$ is the radius of the sphere,

$E$ is the total energy of sound in the medium,

$\mathrm{F}$ is the coefficient of relative density,

$\mathrm{p}_{\mathrm{s}}$ is the density of the sphere.

Taking into account this force, there is no need for the reflector and the emitter to be perpendicular to the vertical axis. As long as the angle relative to the axis does not exceed certain values, the object will still levitate if the force of gravity does not exceed the sum of the main and lateral forces [4].

\section{Matlab software environment for acoustic field control}

It is necessary to explore the possibilities of the package of tools for systems with phased arrays in Matlab to solve the set goals and objectives of the study.

First of all, we need to consider the process of creating planar arrays for the acoustic levitation device. In the Listing 1, we'll look at forming a planar lattice. So, first, we need to determine the number of lattice elements in each row and each column. For a given levitation device, it is sufficient to form a $16 * 16$ lattice in which ultrasound sensors are located. 
Furthermore, we need to set the distance between the elements (emitters) also in each row and column. Finally, we obtain 256 elements, as shown in Figure 1.
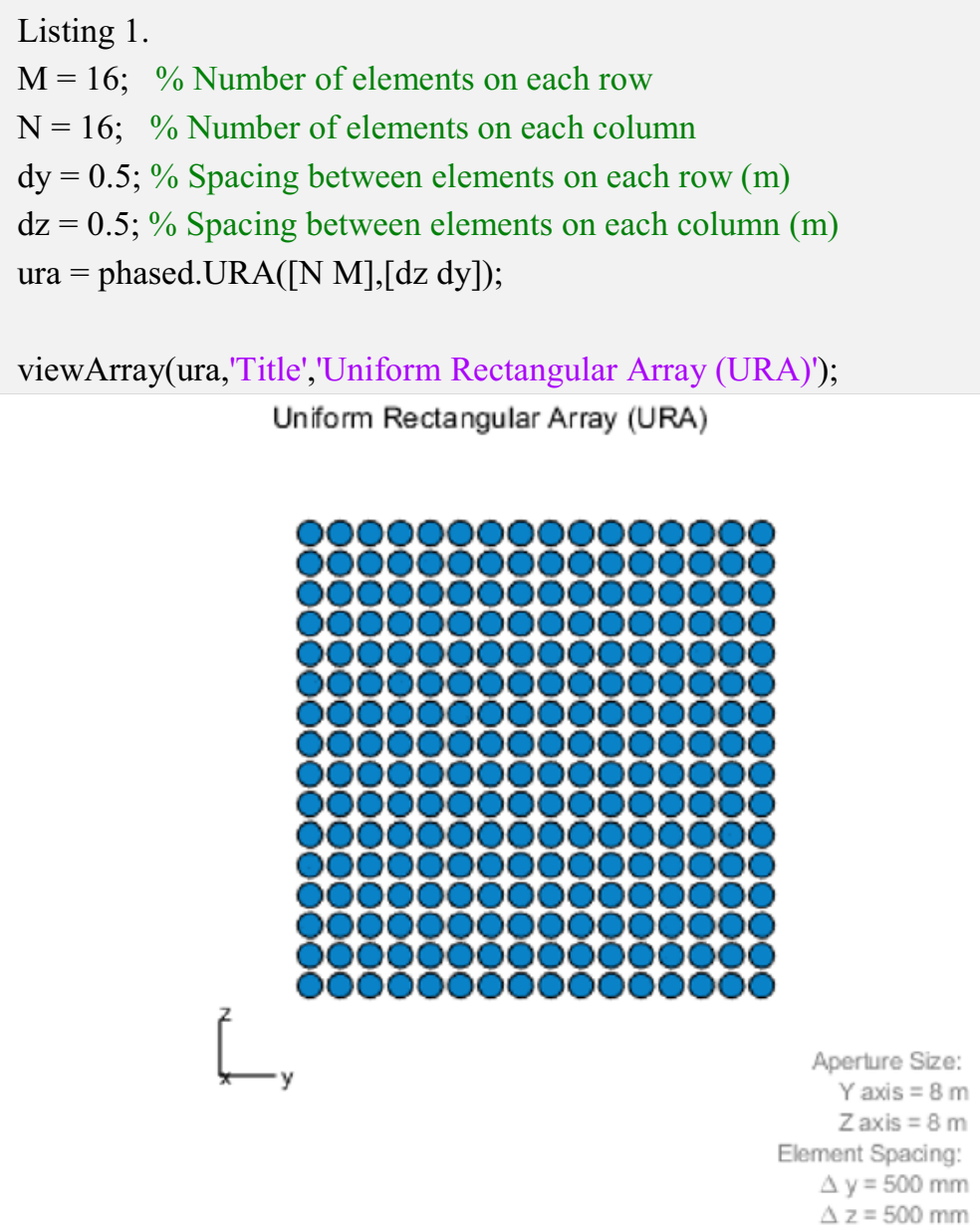

Fig. 1. Planar array with emitters.

Figure 2 shows a graph of amplitude perturbations for a 16x16 radiator array. It reflects the dependence of the normalized power distribution on the voltage value. In this case, the perturbations are taken as statistically independent Gaussian random variables [5-7]. Here the relationship between the ideal amplitude and with the perturbations is noted.

Moreover, figure 2 shows the amplitude narrowing as an indicator of the increase (amplification) of perturbations (distortions).

According to these graphs, it is possible to compare the resonance for all 256 ultrasound sensors (amplitude narrowing) for the sensors in a row with perturbations to the sensors in the "ideal" row of response to perturbations. It is possible to compare the amplitude of the resonance with perturbations to the ideal amplitude (the "failure" elements are exceeded according to the Gaussian variables). 

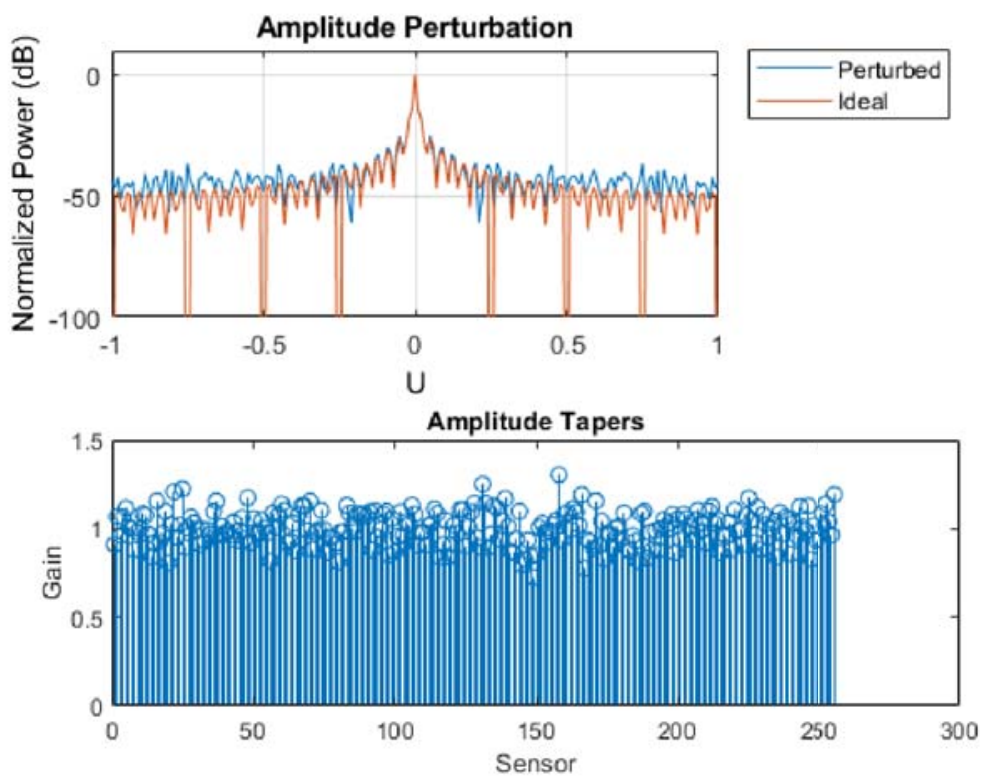

Fig. 2. Amplitude perturbations and decaying field distribution.

Figure 3 shows the addition of phase perturbations according to the algorithm of the first part. Thus, only when narrowing, we have a scale of 1 and a phase shift of 0 (as in the above examples, here random numbers equal to one were also distributed).

It is possible not only to analyze the relationship between the ideal phase amplitude and with perturbations [8], but also to trace the resonance. The resonance can help us to see deviations in sensor values (for example, in the range from 100 to 150).
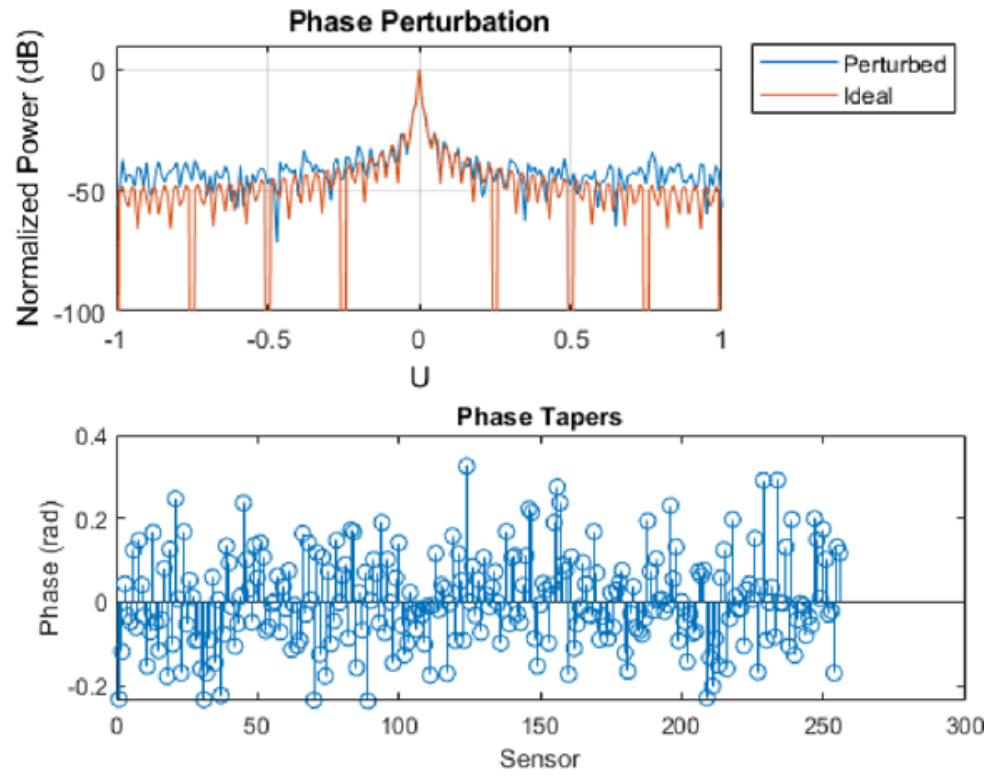

Fig. 3. Phase perturbations and amplitude narrowing. 
We can also show in Figure 4 how to change the position of each sensor with perturbations along three axes (directions). Thereby, it is possible to rebuild the row. It shows the dependence of power on voltage (at positional perturbations).

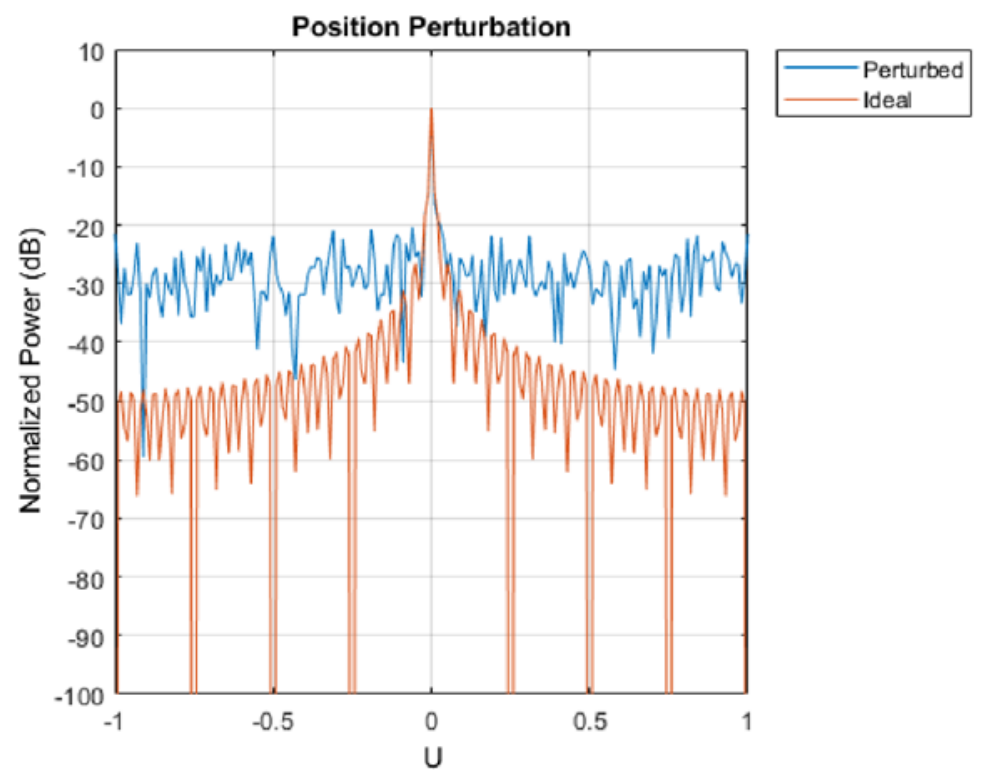

Fig. 4. Positional perturbations.

Next, we will look at the perturbation model in Figure 5. The models with an isotropic characteristic and with perturbations are shown. Thus, it is possible to compare the resonance for two cases with perturbations and without (in an isotropic medium). It can be concluded that in the isotropic model, the power characteristic has better performance compared to the second model.
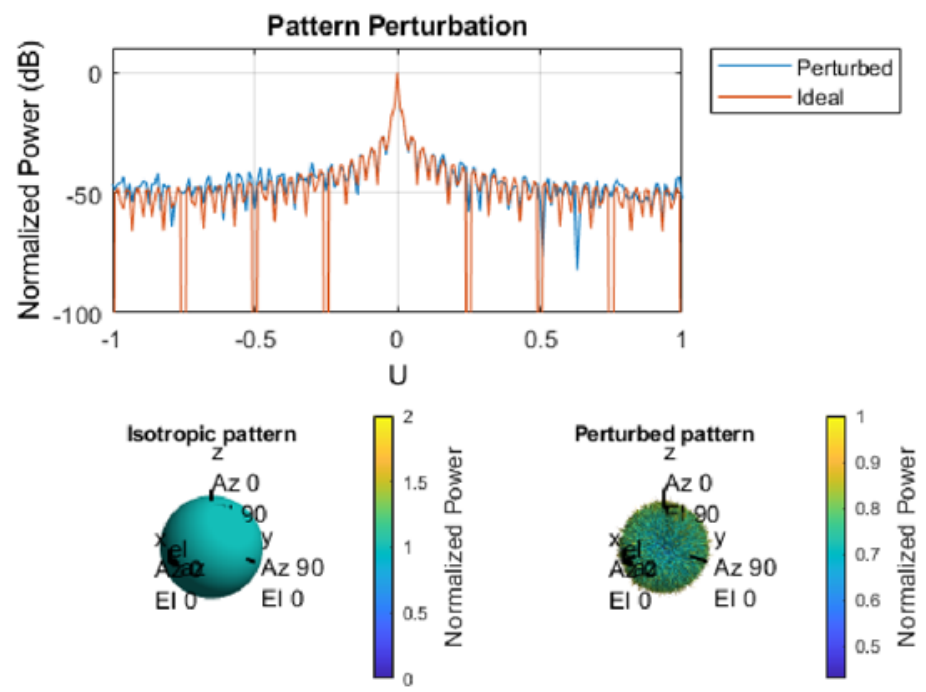

Fig. 5. Pattern perturbations.

Failure of some elements of the 16x16 piezoelectric emitter array is shown in Figure 6. Each of the 256 emitters has a $10 \%$ chance of failure $[9,10,12]$. The graph shows the 
dependence of the normalized power on voltage and also displays graphs with and without failure during the emitters operation. We should focus on how difficult it is to get zero for a number of failing elements, as shown in the graph for a 16x16 array.

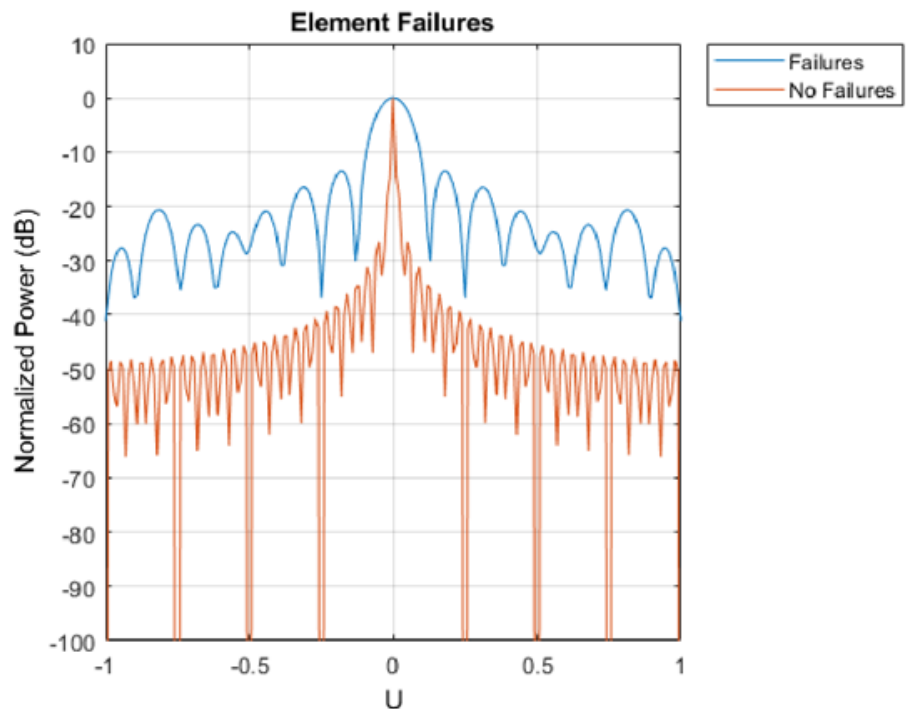

Fig. 6. Pattern perturbations.

As a result, Figure 7 shows the required array with 16x16 ultrasonic emitters with element failures.

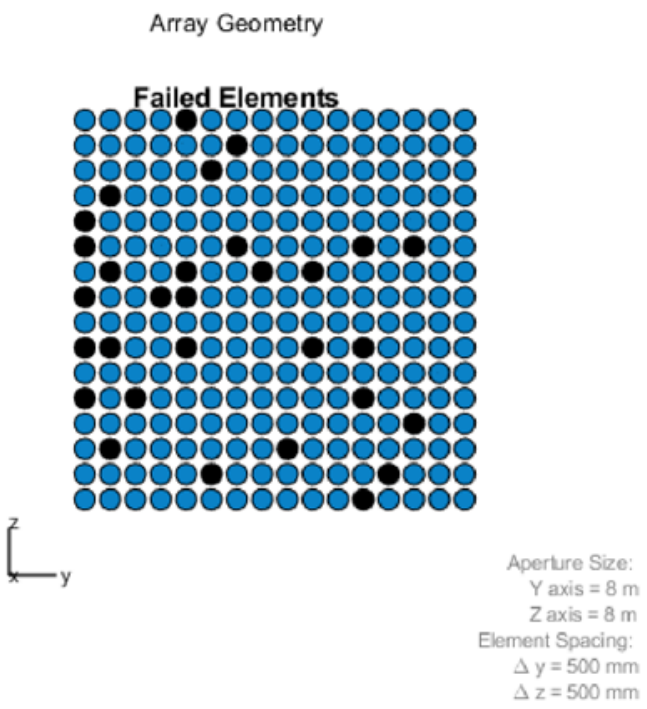

Fig. 7. Image of a row with element failures.

Summing up and given the results, it should be concluded that we received responses for all elements of the array, simulations were carried out for all cases of perturbations, and the effect of the response (resonance) for all cases was considered. The last Figure 7 shows a display of element failures when generating ultrasonic vibrations with a given frequency for 256 array elements. 


\section{Modeling an acoustic field in Comsol Multiphysics}

In this paragraph, it is necessary to analyze the simulation of the ultrasonic levitation sensor. A model from Comsol multiphysics was chosen as a template. Next, let's analyze the graphs of this model. In this software solution we can also consider the material and geometry of the intended product and perform the necessary physical and mathematical calculations.

Figure 8 shows the analysis of the dependence of the distance on the pressure of the acoustic field. According to the results of observations, it can be concluded that the pressure decreases with increasing distance. That's the way it should be when tested in a real environment [11].

In Figure 9, we can see the frequency domain and calculate it using formulas (5.6). Consequently, we can consider the acoustic pressure as a function of frequency and also see in the sum the calculation of the total acoustic pressure with ranking by zones of the ultrasound sensor.

$$
\begin{gathered}
\frac{1}{p c^{2}} \frac{d p_{t}}{d t}+\nabla * u_{t}=Q_{m} \\
p \frac{d u_{t}}{d t}+\nabla *\left(p_{t} I\right)=q_{d}
\end{gathered}
$$

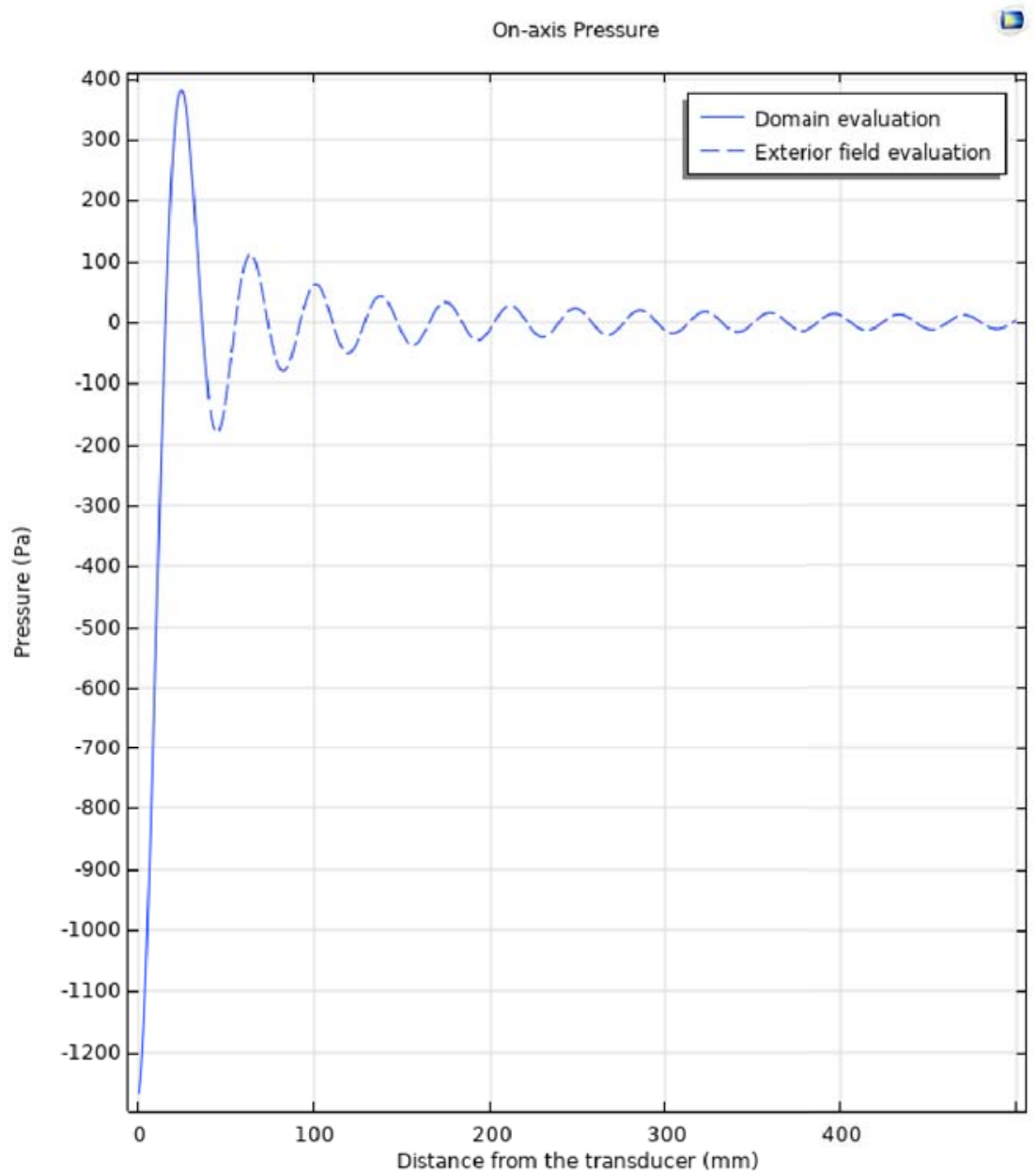

Fig. 8. Graph of the dependence of distance on pressure. 


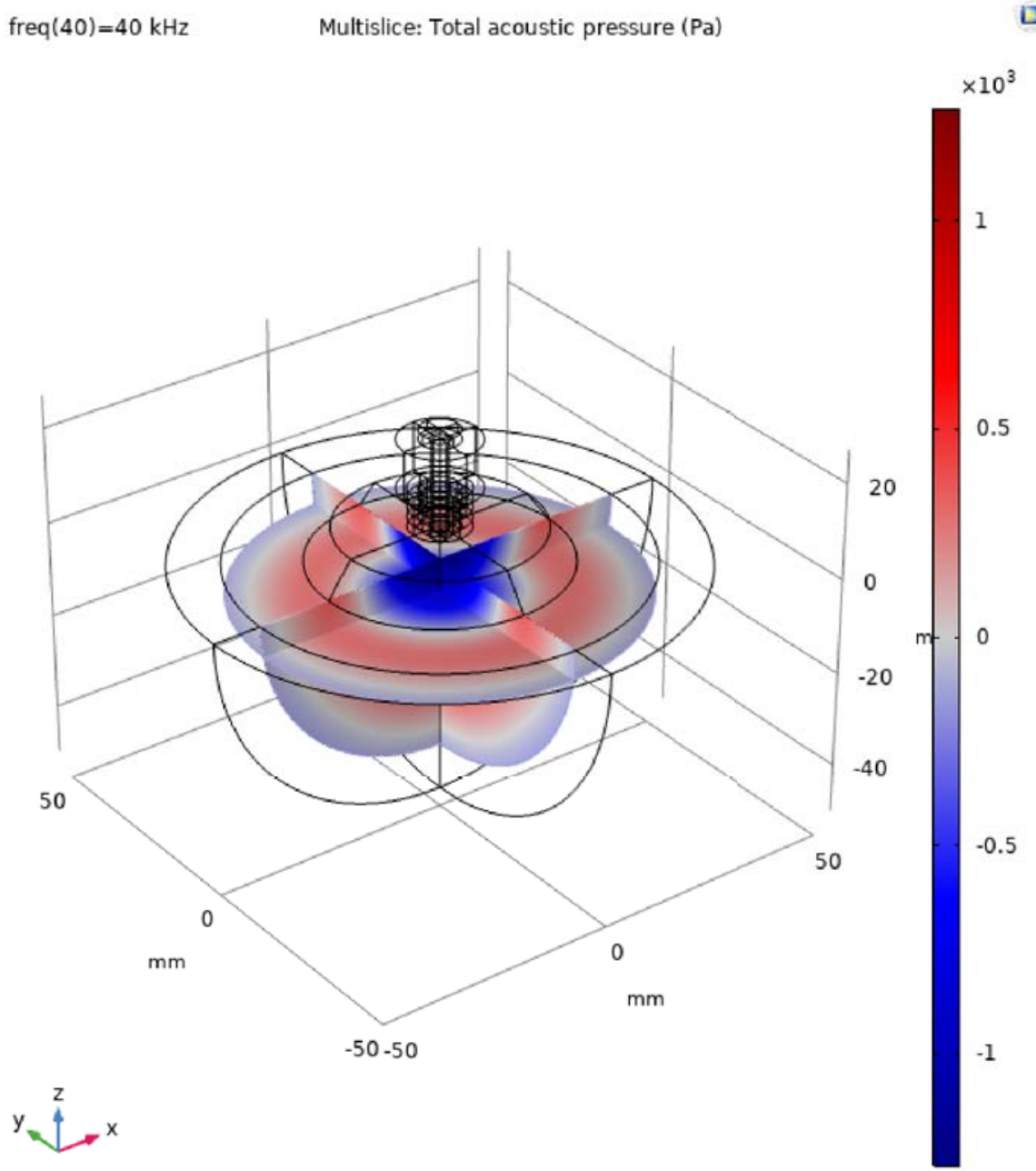

Fig. 9. Total acoustic pressure.

\section{Conclusion}

To sum up, we can say that we managed to explore the capabilities of the software packages for calculating and modeling acoustic levitation systems (and calculating and controlling the acoustic levitation field).

Thus, in the Matlab environment, we can also add a control system that will directly control the operation of the acoustic levitation device.

Besides, in the Comsol environment, one can fully implement the calculations of acoustic systems of different sizes in terms of parameters, using the theory of acoustic field control and integrate into the Matlab environment.

The creation of a control system can be implemented directly in the Matlab environment (however, it is limited in basic calculation systems. 
The integration of the two packages will allow us to create the basis for creating a levitation acoustic field control system.

\section{References}

1. A. Leigh, J. Ronald, Institution of Electrical Engineers (2004)

2. A.I. Borovkov, L.B. Maslov, K.S. Ivanov, E.N. Kovaleva, F.D. Tarasenko, M.A. Zhmaylo, IOP Conf. Ser.: Mater. Sci. Eng. 986, 012033 (2020)

3. A. Al-Dulaimi, S. Zabihi, A. Asif, A. Mohammadi, Comput. Ind. 108, 186-96 (2019)

4. A.A. Zhilenkov, I.R. Epifantsev, IEEE Conference of Russian Young Researchers in Electrical and Electronic Engineering (EIConRus) 1036 (2018) doi: 10.1109/EIConRus.2018.8317266

5. A.V. Ivanov, A.A. Zhilenkov, IEEE Conference of Russian Young Researchers in Electrical and Electronic Engineering (EIConRus) $\mathbf{8 8 2}$ (2018) doi: 10.1109/EIConRus.2018.8317229

6. A.A. Zhilenkov, IEEE Conference of Russian Young Researchers in Electrical and Electronic Engineering (EIConRus) 400 (2018) doi: 10.1109/EIConRus.2018.8317117

7. D. Vuksanović, J. Ugarak, D. Korčok, Sinteza 2016 - International Scientific Conference on ICT and E-Business Related Research, 293-298 (2016)

8. A.D. Karpov, A.A. Zhilenkov, D. Lisitsa, IEEE Conference of Russian Young Researchers in Electrical and Electronic Engineering (EIConRus) 887 (2017) doi: 10.1109/EIConRus.2017.7910697

9. A.A. Zhilenkov, D. Denk, IEEE Conference of Russian Young Researchers in Electrical and Electronic Engineering (EIConRus) $1104 \quad$ (2017) doi: 10.1109/EIConRus.2017.7910748

10. A.A. Zhilenkov, D. Denk, IEEE Conference of Russian Young Researchers in Electrical and Electronic Engineering (EIConRus) $\mathbf{1 1 0 0}$ (2017) doi: 10.1109/EIConRus.2017.7910747

11. D.O. Solovev et al, IOP Conf. Ser. Mater. Sci. Eng. 747, 012076 (2020)

12. B. Dang, A. Zhilenkov, AIP Conf. Proc. 2034, 020007 (2018) https://doi.org/10.1063/1.5067350 